\title{
Upper bounds for arithmetic-geometric index of graphs
}

\section{I. Ž. Milovanović, M. M. Matejić, E. I. Milovanović}

\begin{abstract}
Let $G=(V, E), V=\{1,2, \ldots, n\}$, be a simple connected graph with $n$ vertices and $m$ edges and let $d_{1} \geq d_{2} \geq \cdots \geq d_{n}>0$, be the sequence of vertex degrees. With $i \sim j$ we denote the adjacency of the vertices $i$ and $j$ in graph $G$. With $A G=\sum_{i \sim j} \frac{d_{i}+d_{j}}{2 \sqrt{d_{i} d_{j}}}$ we denote arithmetic-geometric topological index. In this paper we give some new upper bounds for this topological index.
\end{abstract}

Keywords: Arithmetic-geometric index, Zagreb indices, multiplicative Zagreb indices.

\section{Introduction}

Let $G=(V, E), V=\{1,2, \ldots, n\}, E=\left\{e_{1}, e_{2}, \ldots, e_{m}\right\}$, be a simple connected graph with $n$ vertices and $m$ edges, and let $d_{1} \geq d_{2} \geq \cdots \geq d_{n}>0, d_{i}=d(i)$, and $d\left(e_{1}\right) \geq d\left(e_{2}\right) \geq \cdots \geq$ $d\left(e_{m}\right)$, be sequences of its vertex and edge degrees, respectively. We will use the following notation: $\Delta=d_{1}, \delta=d_{n}, \Delta_{e_{1}}=d\left(e_{1}\right)+2$ and $\delta_{e_{1}}=d\left(e_{m}\right)+2$. With $i \sim j$ we denote the adjacency of the vertices $i$ and $j$ in graph $G$.

In [6] and [7] two vertex-degree-based topological indices, the first and the second Zagreb indices, $M_{1}$ and $M_{2}$, were defined as

$$
M_{1}=M_{1}(G)=\sum_{i=1}^{n} d_{i}^{2} \quad \text { and } \quad M_{2}=M_{2}(G)=\sum_{i \sim j} d_{i} d_{j} .
$$

Multiplicative variants of the Zagreb indices, the first and the second multiplicative Zagreb indices, $\Pi_{1}$ and $\Pi_{2}$, are defined in [17] as

$$
\Pi_{1}=\Pi_{1}(G)=\prod_{i=1}^{n} d_{i}^{2} \quad \text { and } \quad \Pi_{2}=\Pi_{2}(G)=\prod_{i \sim j} d_{i} d_{j}
$$

Manuscript received November 12 2017; accepted May 14, 2018.

I. Ž. Milovanović, M. M. Matejić, E. I. Milovanović are with the Faculty of Electronic Engineering, Niš, Serbia. 
The first multiplicative sum Zagreb index, $\Pi_{1}^{*}$, was introduced in [5]

$$
\Pi_{1}^{*}=\Pi_{1}^{*}(G)=\prod_{i \sim j}\left(d_{i}+d_{j}\right)
$$

In [12] connectivity index, $R$, later called Randić index, was defined as

$$
R=R(G)=\sum_{i \sim j} \frac{1}{\sqrt{d_{i} d_{j}}} .
$$

Geometric-arithmetic topological index, GA, was introduced in [18]

$$
G A=G A(G)=\sum_{i \sim j} \frac{2 \sqrt{d_{i} d_{j}}}{d_{i}+d_{j}}
$$

As an inverse variant of this topological index, in [15] arithmetic-geometric vertexdegree-based topological index, $A G$, was defined as

$$
A G=A G(G)=\sum_{i \sim j} \frac{d_{i}+d_{j}}{2 \sqrt{d_{i} d_{j}}} .
$$

In the literature topological index $G A$ was much more studied than $A G$ index, see [2, 3, $4,9,14,16]$. In this paper we are interested in upper bounds on topological index $A G$.

\section{Preliminary results}

In this section we list some analytic inequalities for real number sequences that will be needed in the subsequent considerations.

Let $p=\left(p_{i}\right)$ and $a=\left(a_{i}\right), b=\left(b_{i}\right), i=1,2, \ldots, m$, be positive real number sequences with the properties $0<a \leq a_{i} \leq A<+\infty$ and $0<b \leq b_{i} \leq B<+\infty$. In [1] the following inequality was proven

$$
\left|\sum_{i=1}^{m} p_{i} \sum_{i=1}^{m} p_{i} a_{i} b_{i}-\sum_{i=1}^{m} p_{i} a_{i} \sum_{i=1}^{m} p_{i} b_{i}\right| \leq \frac{1}{4}(A-a)(B-b)\left(\sum_{i=1}^{m} p_{i}\right)^{2} .
$$

Let $a=\left(a_{i}\right), i=1,2, \ldots, m$, be a positive real number sequence. In [8] (see also [19]) the following inequality was proven

$$
\left(\sum_{i=1}^{m} \sqrt{a_{i}}\right)^{2} \leq(m-1) \sum_{i=1}^{m} a_{i}+m\left(\prod_{i=1}^{m} a_{i}\right)^{\frac{1}{m}} .
$$


Let $a=\left(a_{i}\right), i=1,2, \ldots, m$, be positive real number sequence. Then, for any real $r$, $r \leq 0$ or $r \geq 1$, holds (see for example [10])

$$
\sum_{i=1}^{m} a_{i}^{r} \geq m^{1-r}\left(\sum_{i=1}^{m} a_{i}\right)^{r}
$$

If $0 \leq r \leq 1$, then opposite inequality in (3) is valid.

The inequality (3) in the literature is known as the Jensen's inequality.

Let $p=\left(p_{i}\right)$ and $a=\left(a_{i}\right), i=1,2, \ldots, m$, be two positive real number sequences with the properties

$$
p_{1}+p_{2}+\cdots+p_{m}=1 \text { and } 0<a \leq a_{i} \leq A<+\infty .
$$

In [13] (see also [11]) the next inequality was proven

$$
\sum_{i=1}^{m} p_{i} a_{i}+a A \sum_{i=1}^{m} \frac{p_{i}}{a_{i}} \leq a+A
$$

\section{Upper bounds for $A G$}

In the following theorem we establish an upper bound for invariant $A G$ in terms of parameters $m, \Delta_{e_{1}}, \delta_{e_{1}}$ and topological index $R$.

Theorem 3.1. Let $G$ be a simple connected graph with $n$ vertices and $m \geq 2$ edges. Then

$$
A G \leq \frac{n m}{2 R}+\frac{1}{8}\left(\sqrt{\Delta_{e_{1}}}-\sqrt{\delta_{e_{1}}}\right)^{2} R .
$$

Equality holds if and only if $G$ is regular or biregular graph.

Proof For $p_{i}:=\frac{1}{\sqrt{d_{i} d_{j}}}, a_{i}=b_{i}:=\sqrt{d_{i}+d_{j}}, A=B=\sqrt{\Delta_{e_{1}}}, a=b=\sqrt{\delta_{e_{1}}}$, where summing is performed over all edges in graph $G$, the inequality (1) becomes

$$
\sum_{i \sim j} \frac{1}{\sqrt{d_{i} d_{j}}} \sum_{i \sim j} \frac{d_{i}+d_{j}}{\sqrt{d_{i} d_{j}}}-\left(\sum_{i \sim j} \frac{\sqrt{d_{i}+d_{j}}}{\sqrt{d_{i} d_{j}}}\right)^{2} \leq \frac{1}{4}\left(\sqrt{\Delta_{e_{1}}}-\sqrt{\delta_{e_{1}}}\right)^{2}\left(\sum_{i \sim j} \frac{1}{\sqrt{d_{i} d_{j}}}\right)^{2},
$$

i.e.

$$
2 R \cdot A G \leq\left(\sum_{i \sim j} \frac{\sqrt{d_{i}+d_{j}}}{\sqrt{d_{i} d_{j}}}\right)^{2}+\frac{1}{4}\left(\sqrt{\Delta_{e_{1}}}-\sqrt{\delta_{e_{1}}}\right)^{2} R^{2}
$$


For $r=2, a_{i}:=\sqrt{\frac{d_{i}+d_{j}}{d_{i} d_{j}}}$, where summing is performed over all edges in graph $G$, the inequality (3) transforms into

$$
\left(\sum_{i \sim j} \sqrt{\frac{d_{i}+d_{j}}{d_{i} d_{j}}}\right)^{2} \leq m \sum_{i \sim j} \frac{d_{i}+d_{j}}{d_{i} d_{j}}=m n
$$

According to this inequality and (6), follows

$$
2 R \cdot A G \leq n m+\frac{1}{4}\left(\sqrt{\Delta_{e_{1}}}-\sqrt{\delta_{e_{1}}}\right)^{2} R^{2},
$$

wherefrom (5) is obtained.

Theorem 3.2. Let $G$ be a simple connected graph with $n$ vertices and $m \geq 2$ edges. Then

$$
A G \leq \frac{1}{2 R}\left(m \frac{\left(\Pi_{1}^{*}\right)^{\frac{1}{m}}}{\left(\Pi_{2}\right)^{\frac{1}{m}}}+n(m-1)\right)+\frac{1}{8}\left(\sqrt{\Delta_{e_{1}}}-\sqrt{\delta_{e_{1}}}\right)^{2} R .
$$

Equality holds if and only if $G$ is regular or biregular graph.

Proof For $a_{i}:=\frac{d_{i}+d_{j}}{d_{i} d_{j}}$, where summing is performed over all edges in graph $G$, the inequality (2) becomes

$$
\left(\sum_{i \sim j} \sqrt{\frac{d_{i}+d_{j}}{d_{i} d_{j}}}\right)^{2} \leq m \frac{\left(\Pi_{1}^{*}\right)^{\frac{1}{m}}}{\left(\Pi_{2}\right)^{\frac{1}{m}}}+n(m-1) .
$$

According to this inequality and (6), we get (7).

Theorem 3.3. Let $G$ be a simple connected graph with $m \geq 2$ edges. Then

$$
A G \leq \frac{\left(\Delta_{e_{1}}+\delta_{e_{1}}\right) R}{2}-\frac{m \Delta_{e_{1}} \delta_{e_{1}}}{2\left(\Pi_{1}^{*}\right)^{\frac{1}{m}}\left(\Pi_{2}\right)^{\frac{1}{2 m}}} .
$$

Equality holds if and only if $G$ is regular or biregular graph.

Proof For $p_{i}:=\frac{1}{R \sqrt{d_{i} d_{j}}}, a_{i}:=d_{i}+d_{j}, A=\Delta_{e_{1}}, a=\delta_{e_{1}}$, where summing is performed over all edges in graph $G$, the inequality (4) becomes

$$
\sum_{i \sim j} \frac{d_{i}+d_{j}}{\sqrt{d_{i} d_{j}}}+\Delta_{e_{1}} \delta_{e_{1}} \sum_{i \sim j} \frac{1}{\sqrt{d_{i} d_{j}}\left(d_{i}+d_{j}\right)} \leq\left(\Delta_{e_{1}}+\delta_{e_{1}}\right) R
$$


i.e.

$$
2 A G+\Delta_{e_{1}} \delta_{e_{1}} \sum_{i \sim j} \frac{1}{\sqrt{d_{i} d_{j}}\left(d_{i}+d_{j}\right)} \leq\left(\Delta_{e_{1}}+\delta_{e_{1}}\right) R .
$$

Using the arithmetic-geometric mean inequality for real numbers (see e.g. [11]), we get

$$
\sum_{i \sim j} \frac{1}{\sqrt{d_{i} d_{j}}\left(d_{i}+d_{j}\right)} \geq \frac{m}{\left(\Pi_{1}^{*}\right)^{\frac{1}{m}}\left(\Pi_{2}\right)^{\frac{1}{2 m}}} .
$$

From this inequality and the inequality (9), we arrive at (8).

In the following theorem we determine upper bound for invariant $A G$ depending on the parameters $m, \Delta$ and $\delta$.

Theorem 3.4. Let $G$ be a simple connected graph with $m \geq 1$ edges. Then

$$
A G \leq \frac{m}{2}\left(\sqrt{\frac{\Delta}{\delta}}+\sqrt{\frac{\delta}{\Delta}}\right) .
$$

Equality holds if and only if $G$ is regular or biregular graph.

Proof Since

$$
\frac{d_{i}+d_{j}}{\sqrt{d_{i} d_{j}}}=\sqrt{\frac{d_{i}}{d_{j}}}+\sqrt{\frac{d_{j}}{d_{i}}} \leq \sqrt{\frac{\Delta}{\delta}}+\sqrt{\frac{\delta}{\Delta}},
$$

for any edge in graph $G$, it follows

$$
A G=\sum_{i \sim j} \frac{d_{i}+d_{j}}{2 \sqrt{d_{i} d_{j}}} \leq \frac{m}{2}\left(\sqrt{\frac{\Delta}{\delta}}+\sqrt{\frac{\delta}{\Delta}}\right),
$$

which completes the proof.

\section{References}

[1] D. AndricA, C. BADEA, Grüss' inequality for positive linear functionals, Period. Math. Hungar., 19 (1988), 155-167.

[2] K. C. DAS, N. TRINAJSTIĆ, Comparison between first geometric-arithmetic index and atombond connectivity index, Chem. Phys. Lett., 497 (1-3) (2010), 149-151.

[3] K. CH. DAS, On geometric-arithmetic index of graphs, MATCH Commun. Math. Comput. Chem., 64 (2010), 619-630. 
[4] K. C. Das, I. Gutman, B. Furtula, Survey on geometric-arithmetic indices of graphs, MATCH Commun. Math. Comput. Chem., 65 (2011), 595-644.

[5] M. Eliasi, A. Iranmanesh, I. Gutman, Multiplicative versions of first Zagreb index, MATCH Commun. Math. Comput. Chem., 68 (1) (2012), 217-230.

[6] I. Gutman, N. Trinajstić, Graph theory and molecular orbitals. Total $\pi$-electron energy of alternant hydrocarbons, Chem. Phys. Lett., 17 (1972), 535-538.

[7] I. Gutman, B. Ruščić, N. TRinajstić, C. F. Wilcox Graph theory and molecular orbitals. XII. Acyclic polyenes, J. Chem. Phys., 62 (1975), 3399-3405.

[8] H. Kober, On the arithmetic and geometric means and on Hölder's inequality, Proc. Amer. Math. Soc., 9 (1958), 452-459.

[9] I. Ž. Milovanović, E. I. Milovanović, M. M. Matejić, On upper bounds for the geometric-arithmetic topological index, MATCH Commun. Math. Comput. Chem., in press.

[10] D. S. Mitrinović, J. E. PeČARIĆ, A. M. FInK, Classical and new inequalities in analysis, Springer, Netherlands, 1993.

[11] D. S. Mitrinović, P. M. Vasić, Analytic inequalities, Springer Verlag, Berlin-HeidelbergNew York, 1970.

[12] M. Randić, On characterization of molecular branching, J. Am. Chem. Soc., 97 (1975), 6609-6615.

[13] B. C. RenNIE, On a class of inequalities, J. Austral. Math. Soc., 3 (1963), 442-448.

[14] J. M. Rodriguez, J. M. Sigarreta, On the geometric-arithmetic index, MATCH Commun. Math. Comput. Chem., 74 (2015), 103-120.

[15] V. S. Shegehalli, R. Kanabur, Arithmetic-geometric indices of path graph, J. Comput. Math. Sci., 16 (1) (2015), 19-24.

[16] J. M. SigarRetA, Bounds for the geometric-arithmetic index of a graph, Miskolc Math. Notes, 16 (2) (2015), 1199-1212.

[17] R. TODESCHINI, V. CONSONNI, New local vertex invariants and molecular descriptors based on functions of the vertex degrees, MATCH Commun. Math. Comput. Chem., 64 (2) (2010), 359-372.

[18] D. VukičEvić, B. Furtula, Topological index based on the ratios of geometrical and arithmetical means of end-vertex degrees of edges, J. Math. Chem., 46 (2009), 1369-1376.

[19] B. Zhou, I. Gutman, T. Aleksić, A note on the Laplacian energy of graphs, MATCH Commun. Math. Comput. Chem., 60 (2008), 441-446. 